\title{
Homonegativity, Substance Use, Sexual Risk Behaviors, and HIV Status in Poor and Ethnic Men Who Have Sex with Men in Los Angeles
}

\author{
Steven Shoptaw, Robert E. Weiss, Brett Munjas, \\ Christopher Hucks-Ortiz, Sean D. Young, Sherry Larkins, \\ Gregory D. Victorianne, and Pamina M. Gorbach
}

\begin{abstract}
This study evaluates associations between internalized homonegativity and demographic factors, drug use behaviors, sexual risk behaviors, and HIV status among men who have sex with men (MSM) and with men and women (MSM/W). Participants were recruited in Los Angeles County using respondent-driven sampling (RDS) and completed the Internalized Homonegativity Inventory (IHNI) and questionnaires on demographic and behavioral factors. Biological samples were tested for HIV and for recent cocaine, methamphetamine, and heroin use. The 722 MSM and MSM/W participants were predominantly African American (44\%) and Hispanic (28\%), unemployed (82\%), homeless (50\%), and HIV positive (48\%) who used drugs in the past 6 months (79.5\%). Total and Personal Homonegativity, Gay Affirmation, and Morality of Homosexuality IHNI scores were significantly higher for African American men than for other ethnicities, for MSM/W than for MSM, for recent cocaine users than for recent methamphetamine users, and for HIV-seronegative men than for HIVseropositive men. Linear regression showed the Gay Affirmation scale significantly and inversely correlated with the number of sexual partners when controlling for effects of ethnicity/race and sexual identification, particularly for men who self-identified as straight. Highest IHNI scores were observed in a small group of MSM/W $(n=62)$ who never tested for HIV. Of these, $26 \%$ tested HIV positive. Findings describe ways in which internalized homophobia is a barrier to HIV testing and associated HIV infection and signal distinctions among participants in this sample that can inform targeted HIV prevention efforts aimed at increasing HIV testing.
\end{abstract}

KEYWORDS Homophobia, Homonegativity, Drug abuse, Gay men, Bisexual men, HIV

\section{INTRODUCTION}

Incident HIV cases (i.e., recent infection) and new AIDS diagnoses are disproportionately among African Americans and Latinos in Los Angeles County ${ }^{1}$. The Los Angeles AIDS epidemic primarily involves men who have sex with men (MSM) and men who have sex with men and women (MSM/W), groups who account for $76.1 \%$

Shoptaw, Hucks-Ortiz, Larkins, and Victorianne are with the David Geffen School of Medicine at UCLA, Los Angeles, CA, USA; Shoptaw and Larkins are with the Department of Psychiatry and Biobehavioral Sciences, David Geffen School of Medicine at UCLA, Los Angeles, CA, USA; Weiss and Munjas are with the Department of Biostatistics, UCLA School of Public Health, Los Angeles, CA, USA; Young is with the Division of Infectious Disease, David Geffen School of Medicine at UCLA, Los Angeles, CA, USA; Gorbach is with the Department of Epidemiology, UCLA School of Public Health, Los Angeles, CA, USA. Correspondence: Steven Shoptaw, David Geffen School of Medicine at UCLA, Los Angeles, CA, USA. (E-mail: sshoptaw@mednet.ucla.edu) 
of all AIDS cases. Increasing numbers of women with AIDS, particularly African Americans and Latinas, have been reported, which raises questions about the direction of the epidemic ${ }^{1}$. Factors that might promote HIV moving from a concentrated epidemic involving MSM and MSM/W to a more generalized epidemic involving women remain poorly specified. One factor that may contribute is highrisk sexual behavior of men with both male and female sexual partners driven by internalized homonegativity ${ }^{2,3}$.

Internalized homonegativity (or homophobia) reflects a lack of positive beliefs about being gay, about valuation of the larger gay community, and about the morality of being gay ${ }^{2}$. In MSM and MSM/W, internalized homophobia correlates with low selfesteem and high-risk sex ${ }^{4}$. By contrast, levels of internalized homophobia inversely correspond with sexual satisfaction ratings among White $\mathrm{MSM}^{5}$. Some have speculated that MSM with high levels of internalized homophobia are not exposed to cultural norms and corresponding HIV-risk reduction messages that reinforce safer sexual behaviors ${ }^{6}$. Recent data show that MSM/W, however, restrict unprotected sex solely to regular/main male or female partners ${ }^{7}$. The men presumably are more likely to know the HIV status of these regular/main partners and consequently limit unprotected sex with other men or women in response to perceived messages regarding HIV risks associated with unprotected sex with status unknown partners ${ }^{7}$. Higher scores of internalized homophobia have been associated with higher expectations that substance use during sex would enhance sexual experiences, both factors that associate with practice of unprotected anal intercourse (UAI) ${ }^{8}$.

Internalized homonegativity and sexual risk behaviors are factors that may be strongly correlated in communities of MSM and MSM/W of color; experiences with internalized homophobia are thought to be particularly profound and involve incorporation of disapproval of MSM from both minority and dominant cultures ${ }^{9}$. Some MSM and MSM/W of color may resist or even reject adoption of a sexual identity that recognizes their experiences with other men and instead nurture a heterosexual identity ${ }^{10}$. Long-term consequences to maintaining a heterosexual identity while engaging in same sex behaviors include high-risk sex, substance abuse, psychological distress, and negative physical health effects, including HIV ${ }^{11}$.

In turn, substance abuse is significantly associated with levels of internalized homonegativity and UAI. Use of stimulants and other drugs cover feelings of shame and internalized homonegativity to facilitate sexual behaviors for MSM and MSM/W that are not possible when not under the influence ${ }^{12,13}$. Prior studies show that MSM, including men of color, use substances to facilitate $\operatorname{sex}^{14}$, though African American MSM use significantly less substances than White MSM for this purpose ${ }^{15}$.

This study evaluates associations between reported demographic factors, drug use behaviors, sexual risk behaviors, HIV status, and levels of internalized homonegativity among MSM and MSM/W who are poor, urban, and of ethnic descent in Los Angeles.

\section{METHODS}

Participants. Participants were MSM or MSM/W who participated in one of the two waves of data collection (2005-2006; 2006-2008) at the Los Angeles site for NIDA's Sexual Acquisition and Transmission of HIV-Cooperative Agreement Program (SATH-CAP). Participants were: (1) over 18 years of age; (2) MSM or MSM/W who engaged in the past 6 months in any anal intercourse (AI) and/or a male or female drug user (i.e., self-reported use of powder cocaine, crack cocaine, 
heroin, methamphetamine, or any injection drug use)—or a sexual partner of a SATH-CAP participant. Only MSM and MSM/W are included in this analysis as only they completed the homonegativity questions.

Study Sample. The sample was compiled using respondent-driven sampling ${ }^{16}$. For each of two waves of data collection, "seeds" (individuals willing to participate in the study) were passively recruited via flyers that described the research project, subsequently made an appointment, arrived at the clinic, completed questionnaires and provided biological samples for which they were compensated \$50. Upon completion, participants were provided with coupons to recruit people to the study who they knew to be MSM and/or drug users or who were their sexual partners, with the potential of earning an additional \$140 in vouchers for the referrals (see Iguchi et al., this volume).

Measures. An audio computer-assisted self-interview (ACASI) collected detailed information regarding: (1) drug use-past 6 months use of powder cocaine, crack cocaine, methamphetamine, heroin, and speedballs (heroin plus cocaine); (2) sexual risk behaviors-numbers of male and female sexual partners in the prior 6 months, numbers of specific behaviors engaged in over the past 6 months while having sex with male and female partners (particularly those under the influence of drugs), numbers of specific sexual and drug use behaviors with each of the last three sexual and drug-using partners over the past 6 months; (3) the Internalized Homonegativity Inventory ${ }^{2}$ - a 23 -item questionnaire that yielded a total score and three subscales (Personal Homonegativity, Gay Affirmation, Morality of Homosexuality). Personal Homonegativity included 11 items on feelings of shame, anxiety, and resentment over being homosexual. Gay Affirmation included seven items on pride and acceptance of being homosexual. Morality of Homosexuality included five items on the morality of homosexuality. The total and subscales are compiled using sums; (4) self-reported variables measuring “outness," i.e., the number of men you know who have sex with other men, the number of friends/family who know you have sex with other men; (5) self-reported sexual identification, defined as (a) Gay or homosexual, (b) Bisexual, (c) Straight, (d) Down Low, Same Gender Loving, Messing Around on the Other Team, (e) Male to Female Transgender, (f) No Label; (6) biological specimens-oral HIV rapid test with confirmatory ELISA for positive results, and urine drug screens tested for metabolites of cocaine, methamphetamine, and heroin (opioids). Men in the sample who reported having had sex with other men, but not within the previous 6 months, were categorized as having had "no sex."

Data analysis. Prior to addressing study questions, the pattern of missing data was explored. For cases missing three or fewer items on the IHNI, subject means were imputed for missing items to avoid casewise deletion. This allows retention of a significant number of cases that would otherwise be lost in the analyses. Psychometric properties of the IHNI total and the subscales were assessed using Cronbach's alphas for internal consistency and a principal components factor analysis for latent structure of the data. IHNI scores were correlated with selfreported measures of "outness" to examine content validity.

Chi-square analyses compared the distributions of percentages of MSM and MSM/W among demographic, drug and sex variables. Participants reporting no sex in the previous 6 months who identified as MSM or MSM/W were included separately. ANOVAs tested univariate differences between IHNI scores and interval 
level demographic, drug and sex variables. Hypotheses for condom use in the last three reported sexual partners were fit with a random intercept model in Proc GLIMMIX in SAS version 9.1 (SAS Institute, Cary, NC, USA) and were used to evaluate whether internalized homonegativity scores predicted condom use when controlling for ethnicity/race and sexual identification. Linear regression was used to evaluate the effect of IHNI and subscale scores on the number of reported sexual partners and the number of reported AI partners. When evaluating the effects of IHNI score and subscale scores on sexual risk behaviors, ethnicity/race and self sexual identification were used as covariates. Logistic regression was used to determine the association of demographic, drug use, and sexual risk behaviors with confirmed HIV status.

All scientific and research procedures were overseen by the UCLA Human Subjects Protection Committee and the RAND Institutional Review Board.

\section{RESULTS}

Study Sample. Figure 1 describes sample composition. The final sample of 722 MSM and MSM/W included data from 55 participants who had three or fewer IHNI items missing and were imputed. Participants with three or fewer missing items differed significantly from those with complete data only along biological and self-report of HIV serostatus (both $p<0.001$ ). Participants with imputed IHNI items were more likely to be HIV seronegative than those who completed the survey.

The sample was MSM and MSM/W, predominantly African American (44\%) or Latino $(28 \%)$ men, likely to report drug use in the past 6 months $(79.5 \%)$ and many who are HIV positive $(47.5 \%)$. Biological testing for HIV showed Latino men had the highest HIV prevalence $(64.6 \%)$ followed by White $(43.4 \%)$ and Black $(39.2 \%)$ men. Among the smaller number of men with other ethnicities, $47.7 \%$ tested HIV positive. In the smaller group of men who did not know their HIV status, $26 \%$ (of 66) who reportedly had tested previously but did not know their results and $26 \%$ (of 62) men who reportedly never tested were confirmed HIV positive. Table 1 shows significant associations between MSM and MSM/W by demographic, drug use, sexual behavior, and HIV status characteristics. While $52 \%$ of MSM/W informed their female partners about having sex with men, only $18 \%$ of MSM/W reported informing their male partners that they also have had sex with women.

\begin{tabular}{|c|c|}
\hline $\begin{array}{l}\text { Total Number of Valid Participants: } \\
\text { Wave } 1 \text { Participants Total: } n=797 \\
\text { Wave } 2 \text { Participants Total: } n=755\end{array}$ & $n=1,552$ \\
\hline \multicolumn{2}{|c|}{$\begin{array}{l}\text { Subtract Participants with Exclusion Criteria: } \\
\text { Females: } n=269 \\
\text { Heterosexual men: } n=281 \\
\text { Never Had Sex: } n=3 \\
\text { Female to Male Transgendered: } n=2\end{array}$} \\
\hline \multirow{2}{*}{\multicolumn{2}{|c|}{$\begin{array}{l}\text { Total Valid MSM and MSM/W Participants } \\
\text { Subtract MSM and MSM/W Participants with Missing Data } \\
\text { Coding Error Wave 1: } n=186 \\
\text { More than } 3 \text { IHNI Items Missing: } n=89\end{array}$}} \\
\hline & \\
\hline & $-\quad 275$ \\
\hline Total Participants in These Analyses & $\overline{n=722}$ \\
\hline
\end{tabular}

FIGURE 1. Construction of study sample. 
TABLE 1 Demographic factors, drug use, sexual risk behaviors, and HIV status for male participants who reported having sex with men (MSM) and with men and women (MSM/W) in the past 6 months

\begin{tabular}{|c|c|c|c|c|c|}
\hline & $N$ & No sex (\%) & MSM (\%) & MSM/W (\%) & Chi-sq $(d f), p$ value \\
\hline \multicolumn{6}{|l|}{ Demographic variables } \\
\hline \multicolumn{5}{|l|}{ Age } & \multirow[t]{6}{*}{$x^{2}(8)=22,0.0041$} \\
\hline$<30$ & 84 & 11 & 62 & 27 & \\
\hline $30-39$ & 181 & 13 & 60 & 28 & \\
\hline $40-49$ & 299 & 15 & 53 & 32 & \\
\hline $50-59$ & 122 & 30 & 25 & 45 & \\
\hline$>=60$ & 20 & 16 & 40 & 44 & \\
\hline \multicolumn{5}{|l|}{ Race } & \multirow[t]{5}{*}{$x^{2}(6)=47,<0.0001$} \\
\hline White & 152 & 13 & 57 & 30 & \\
\hline Black & 312 & 15 & 41 & 44 & \\
\hline Hispanic & 198 & 14 & 69 & 17 & \\
\hline Other & 44 & 18 & 48 & 34 & \\
\hline \multicolumn{5}{|l|}{ Education level } & \multirow[t]{4}{*}{$x^{2}(4)=23,0.0001$} \\
\hline Less than high school & 156 & 22 & 47 & 30 & \\
\hline High school & 233 & 15 & 45 & 40 & \\
\hline More than high school & 316 & 11 & 61 & 28 & \\
\hline \multicolumn{5}{|l|}{ Employment status } & \multirow[t]{4}{*}{$x^{2}(4)=15,0.0045$} \\
\hline Unemployed & 581 & 15 & 49 & 35 & \\
\hline Part-time & 56 & 9 & 68 & 23 & \\
\hline Full-time & 69 & 13 & 68 & 19 & \\
\hline \multicolumn{5}{|l|}{ Income in past month (legal) } & \multirow[t]{4}{*}{$x^{2}(4)=23,0.0001$} \\
\hline$\$ 0-\$ 500$ & 424 & 14 & 46 & 39 & \\
\hline$\$ 501-\$ 1,000$ & 171 & 18 & 59 & 23 & \\
\hline$>\$ 1,000$ & 102 & 10 & 66 & 25 & \\
\hline \multicolumn{5}{|l|}{ Homeless in past year } & \multirow[t]{3}{*}{$x^{2}(2)=17,0.0002$} \\
\hline No & 351 & 15 & 60 & 26 & \\
\hline Yes & 355 & 14 & 46 & 40 & \\
\hline \multicolumn{5}{|l|}{ Marital status } & \multirow[t]{5}{*}{$x^{2}(6)=37,<0.0001$} \\
\hline Single & 461 & 16 & 58 & 27 & \\
\hline Married/cohabitating & 70 & 14 & 59 & 27 & \\
\hline Formerly married & 160 & 11 & 36 & 52 & \\
\hline Other & 15 & 20 & 40 & 40 & \\
\hline \multicolumn{6}{|l|}{ Drug use variables } \\
\hline \multicolumn{5}{|l|}{ Inject drugs - past 30 days } & \multirow[t]{3}{*}{$x^{2}(2)=11,0.0051$} \\
\hline No & 572 & 13 & 56 & 31 & \\
\hline Yes & 130 & 18 & 40 & 42 & \\
\hline \multicolumn{5}{|l|}{ Inject drugs_-ever } & \multirow[t]{3}{*}{$x^{2}(2)=5,0.0688$} \\
\hline No & 494 & 15 & 55 & 30 & \\
\hline Yes & 212 & 15 & 47 & 39 & \\
\hline \multicolumn{5}{|l|}{ Urine drug screen, cocaine } & $x^{2}(2)=10,0.0069$ \\
\hline Negative & 580 & 14 & 56 & 31 & \\
\hline Positive & 125 & 18 & 40 & 42 & \\
\hline Urine drug screen, methamphe & amine & & & & $x^{2}(2)=16,0.0004$ \\
\hline Negative & 639 & 14 & 52 & 35 & \\
\hline Positive & 66 & 24 & 64 & 12 & \\
\hline Urine drug screen, heroin & & & & & $x^{2}(2)=7,0.0359$ \\
\hline Negative & 663 & 14 & 53 & 33 & \\
\hline Positive & 42 & 26 & 55 & 19 & \\
\hline Self-report, methamphetamine & last 6 & lonths & & & $x^{2}(2)=0.1,0.9567$ \\
\hline No & 379 & 14 & 53 & 33 & \\
\hline
\end{tabular}


TABLE 1 (continued)

\begin{tabular}{|c|c|c|c|c|c|}
\hline & $N$ & No sex (\%) & MSM (\%) & MSM/W (\%) & Chi-sq $(d f), p$ value \\
\hline Yes & 326 & 15 & 52 & 33 & \\
\hline \multicolumn{5}{|c|}{ Self report, cocaine/crack, last 6 months } & \multirow{3}{*}{$x^{2}(2)=30,<0.0001$} \\
\hline No & 265 & 17 & 63 & 20 & \\
\hline Yes & 440 & 13 & 47 & 40 & \\
\hline \multicolumn{5}{|l|}{ Self-report, heroin, last 6 months } & \multirow{3}{*}{$x^{2}(2)=26,<0.0001$} \\
\hline No & 576 & 14 & 57 & 29 & \\
\hline Yes & 128 & 18 & 33 & 49 & \\
\hline \multicolumn{5}{|c|}{ Self-report, speedball, last 6 months } & \multirow{3}{*}{$x^{2}(2)=27,<0.0001$} \\
\hline No & 597 & 15 & 56 & 29 & \\
\hline Yes & 108 & 14 & 32 & 53 & \\
\hline \multicolumn{5}{|c|}{ Self-report, any drug, last 6 months } & \multirow{3}{*}{$x^{2}(2)=13,0.0016$} \\
\hline No & 144 & 11 & 66 & 23 & \\
\hline Yes & 560 & 16 & 49 & 35 & \\
\hline \multicolumn{6}{|l|}{ Sexual behaviors } \\
\hline \multicolumn{5}{|c|}{ Number of sexual partners—past 6 months } & \multirow[t]{5}{*}{$x^{2}(6)=742,<0.0001$} \\
\hline None & 103 & 100 & 0 & 0 & \\
\hline 1 & 106 & 0 & 85 & 15 & \\
\hline 2 & 127 & 0 & 53 & 47 & \\
\hline 3 or more & 370 & 0 & 58 & 42 & \\
\hline \multicolumn{4}{|l|}{ Condom use last 6 months } & & \multirow{3}{*}{$x^{2}(2)=154,<0.000$} \\
\hline No unprotected sex & 313 & 33 & 47 & 20 & \\
\hline Unprotected sex & 372 & 0 & 56 & 44 & \\
\hline \multicolumn{4}{|l|}{ Sexual identification } & & \multirow[t]{7}{*}{$x^{2}(10)=314,<0.0001$} \\
\hline Gay or homosexual & 321 & 17 & 80 & 3 & \\
\hline Bisexual & 193 & 7 & 27 & 66 & \\
\hline Straight & 60 & 30 & 10 & 60 & \\
\hline $\begin{array}{l}\text { DL, same gender, mess } \\
\text { around }\end{array}$ & 57 & 12 & 30 & 58 & \\
\hline Male $>$ female & 30 & 20 & 77 & 3 & \\
\hline No label & 32 & 13 & 25 & 63 & \\
\hline \multicolumn{4}{|l|}{ Sexual behavior } & & \multirow[t]{6}{*}{$x^{2}(8)=459,<0.0001$} \\
\hline Only with men & 357 & 17 & 82 & 1 & \\
\hline Mostly with men & 108 & 5 & 49 & 46 & \\
\hline Equal with men and women & 51 & 8 & 12 & 80 & \\
\hline Mostly with women & 151 & 9 & 9 & 81 & \\
\hline Only with women & 26 & 65 & 4 & 31 & \\
\hline \multicolumn{5}{|l|}{ HIV status } & \multirow{4}{*}{$x^{2}(2)=161,<0.0001$} \\
\hline Confirmed HIV status & & & & & \\
\hline Negative & 370 & 13 & 33 & 54 & \\
\hline Positive & 335 & 16 & 74 & 10 & \\
\hline \multicolumn{5}{|l|}{ Self-report HIV status } & \multirow[t]{5}{*}{$x^{2}(6)=178,<0.0001$} \\
\hline Test, don't know & 65 & 15 & 55 & 29 & \\
\hline Negative & 283 & 11 & 31 & 58 & \\
\hline Positive & 298 & 15 & 76 & 8 & \\
\hline No test & 59 & 25 & 37 & 37 & \\
\hline
\end{tabular}


Psychometric Properties. IHNI scores in this sample were substantially higher for both the total scores and for all three subscale scores in comparison to the original sample used in test construction (higher scores indicate higher levels of internalized homonegativity). Cronbach's alphas for the IHNI total $(\alpha=0.91)$ and the Personal Homonegativity $(\alpha=0.90)$, Gay Affirmation $(\alpha=0.83)$, and Morality of Homosexuality $(\alpha=0.76)$ scales were in the excellent-to-good range. The factor analysis yielded two factors that confirmed the first two factors (Personal Homonegativity, Gay Affirmation) ${ }^{2}$; the items that did not load on the two large factors were on the Morality of Homosexuality subscale. As there were no statistically significant differences between Waves along IHNI or subscale scores, the data from both samples were combined for analyses.

Table 2 shows means and standard deviations for the IHNI total and the Personal Homonegativity, Gay Affirmation, and Morality of Homosexuality scores by demographic, drug use, sexual behavior, and HIV status characteristics. Univariate analyses showed statistically significant differences by age on the IHNI total, Gay Affirmation, and Morality subscales, with scores increasing with age. Scores for the IHNI total, Gay Affirmation, and Morality scores were significantly higher for African American men than for those of other ethnicities. Higher scores were observed for the IHNI total, Personal Homonegativity, Gay Affirmation, and Morality of Homosexuality scores for participants who attained high school or less educational levels. Significant differences also were found by employment status and income levels for the past month with IHNI total, Gay Affirmation, and Morality of Homosexuality scores being lower among those who reported full-time employment and higher monthly income levels. Homeless men scored significantly higher on the IHNI total score than those who denied homelessness. IHNI total, Gay Affirmation, and Morality of Homosexuality subscales differed significantly by marital status, with higher levels observed for participants who were formerly married.

IHNI scores varied significantly by drug use; injection drug users (lifetime and in the past 30 days) scored significantly higher on the IHNI total and Gay Affirmation scores than those who denied injection drug use. The Personal subscale was significantly higher for lifetime injectors only. The Morality subscale was significantly higher for recent injectors (past 30 days), though not for injectors lifetime. Urine drug screening results for heroin were not associated with IHNI or subscale scores, though self-report of use of heroin and of speedballs (heroin plus cocaine) was significantly associated with increased IHNI total and the Personal, Gay Affirmation, and Morality subscales. Participants who tested positive for cocaine screens were significantly higher on the IHNI total, Gay Affirmation, and Morality subscale scores than those who tested negative. Methamphetamine drug screen results showed opposing patterns with participants who tested positive for methamphetamine being significantly lower on the IHNI total, Personal, Gay Affirmation, and Morality subscales than those who tested methamphetamine negative. Self-report of methamphetamine use was not associated with IHNI scores.

IHNI total and subscale scores were highest for MSM/W, lowest for MSM, and intermediate for the men who reported having no sex (all $p<0.0001$ ). An HIV seropositive test strongly and significantly correlated with lower IHNI total and subscale scores. A similar pattern was observed for participants' self-report of HIV status, with lower scores for those who reported being HIV positive. As expected, IHNI scores were highest for men who self-identified as straight or as any label indicating bisexuality. Lowest IHNI scores were observed among men who identified as gay or homosexual. 


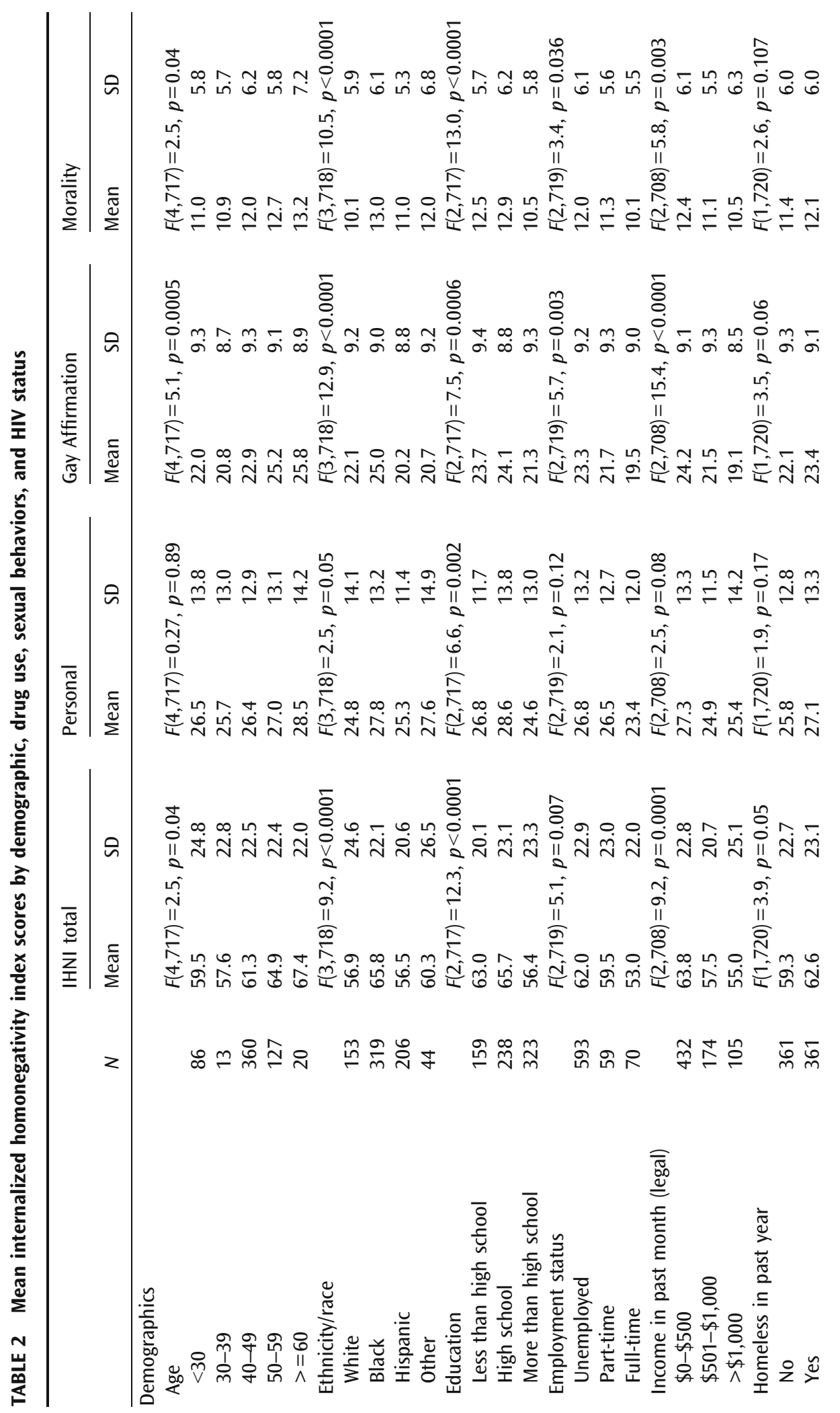




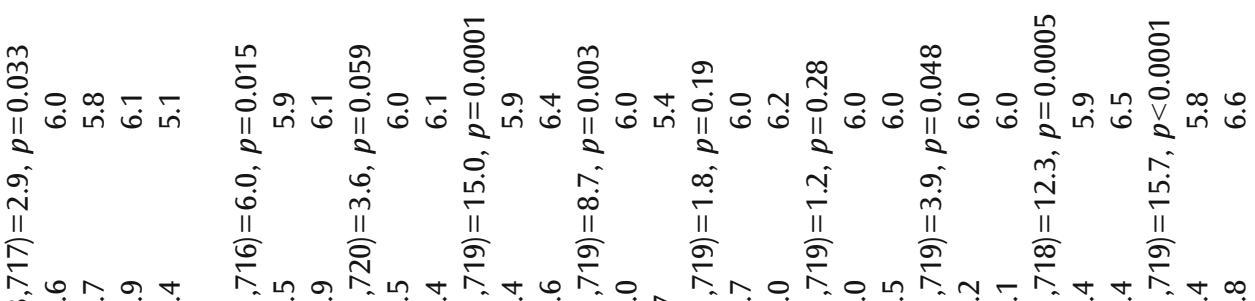

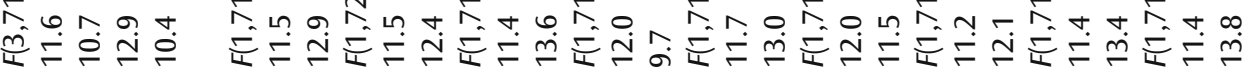

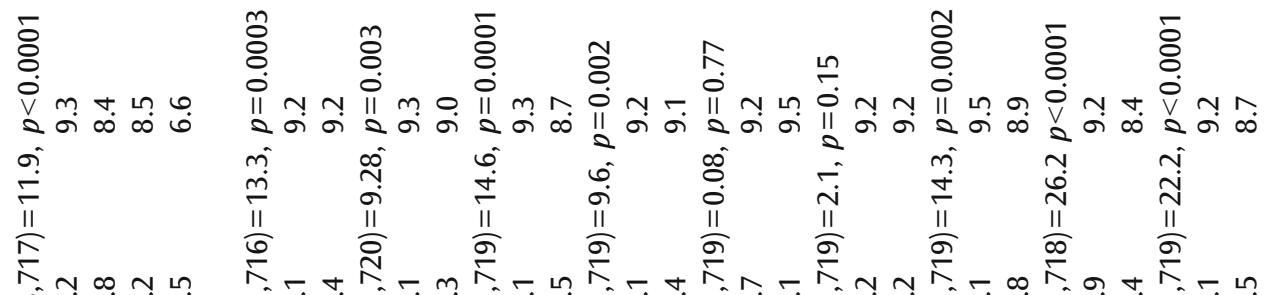

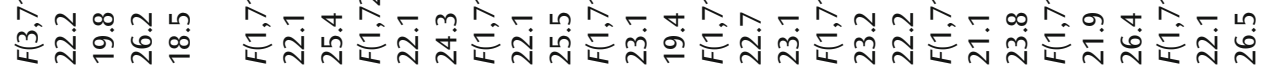

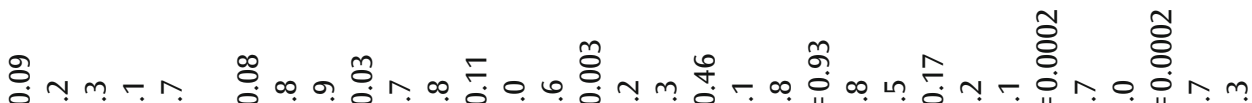

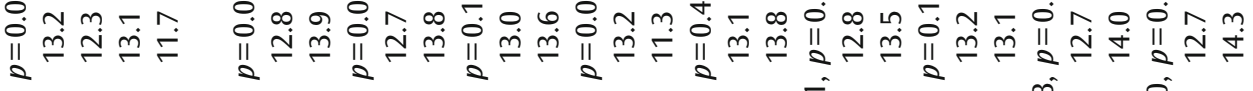

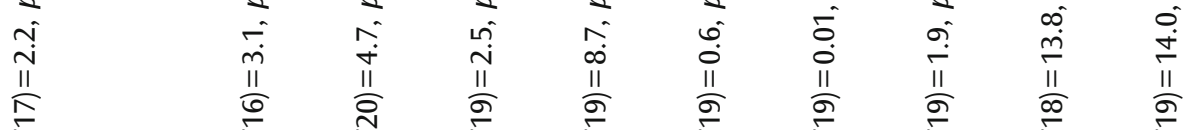

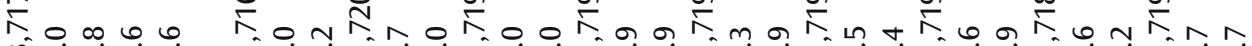

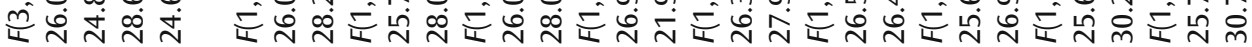

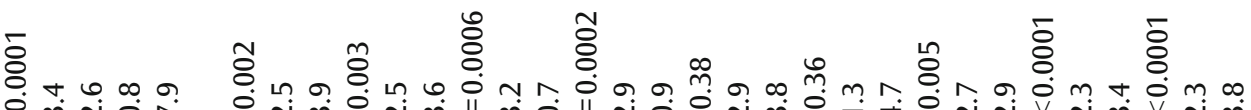
v

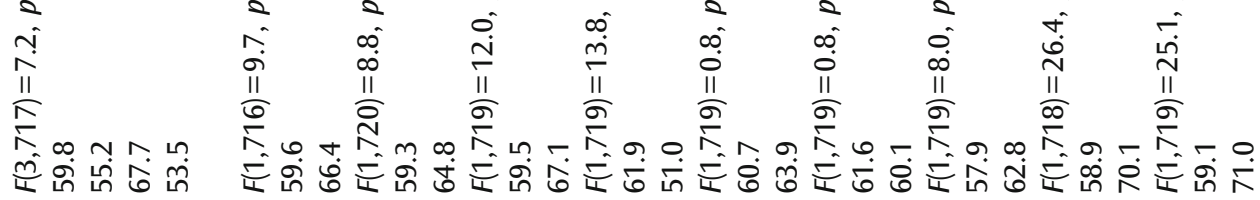

少

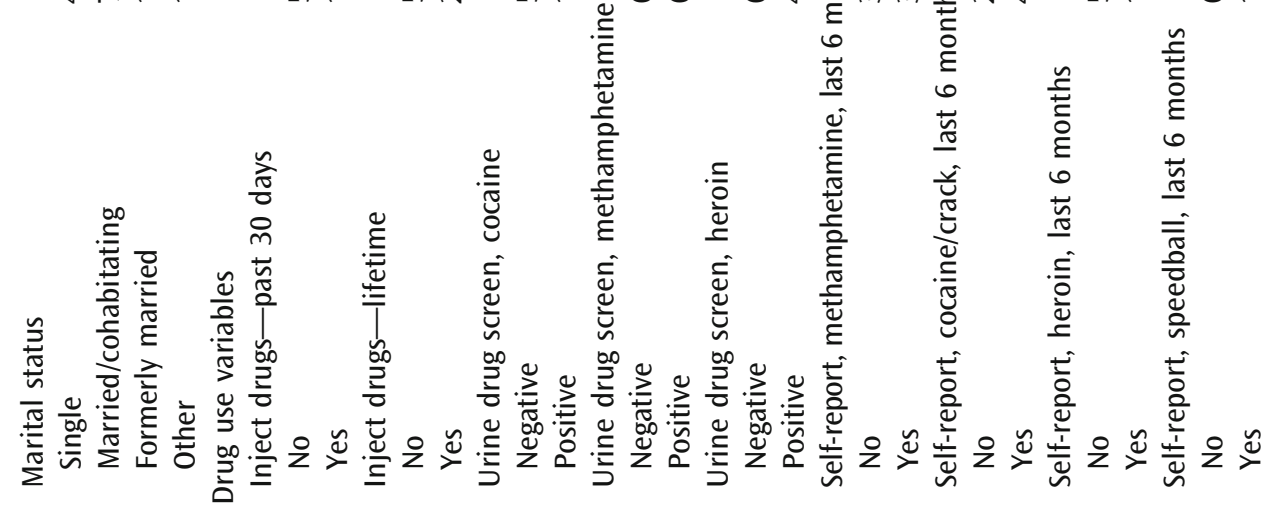




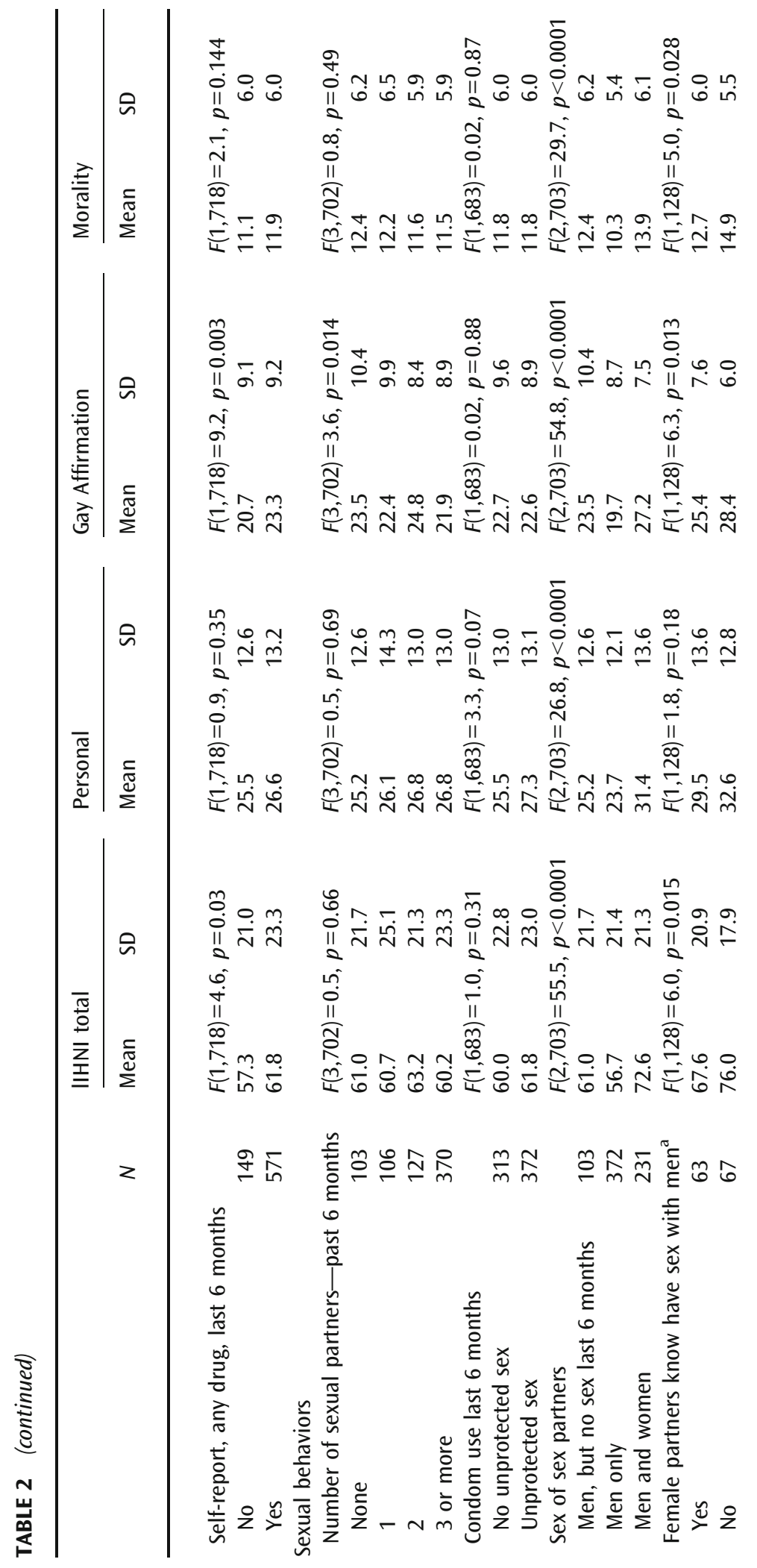




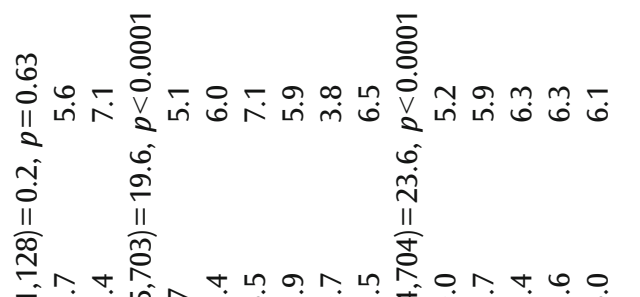

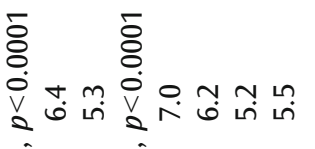

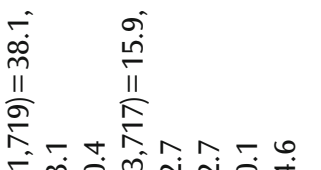

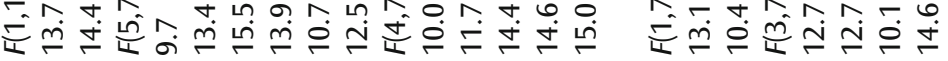

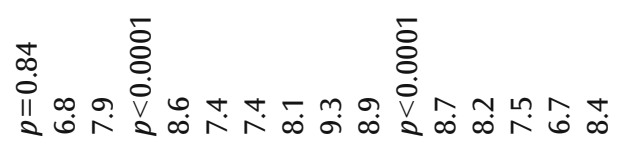

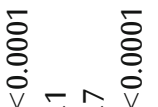

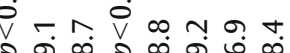

+े

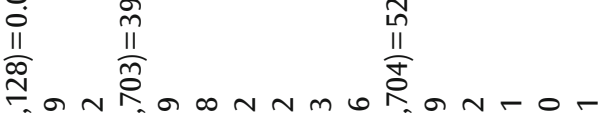

in

กั

$\frac{1}{2} \quad \frac{1}{\sqrt{2}}$

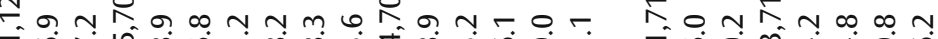

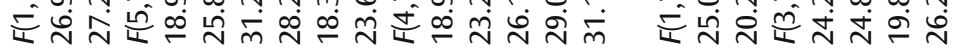

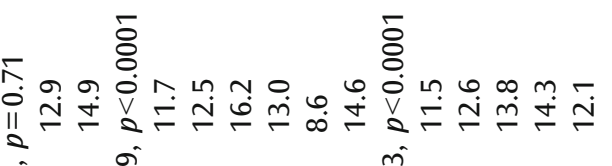

ठิ

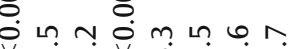

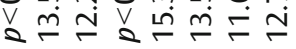

离

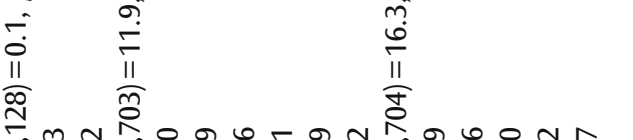

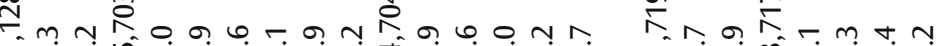

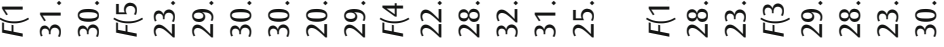

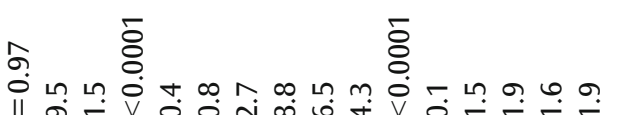

$\bar{\delta} \quad \overline{8}$

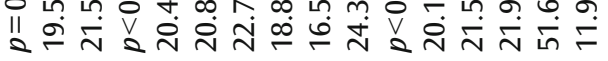

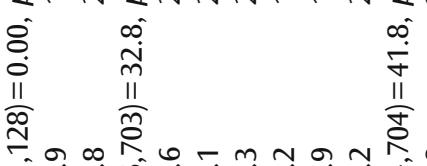

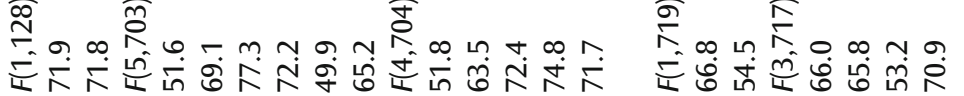

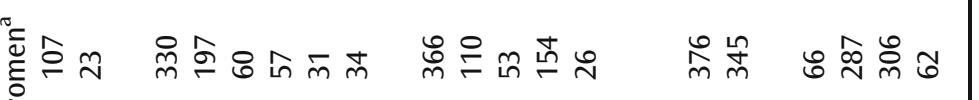

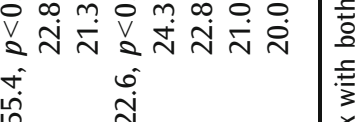

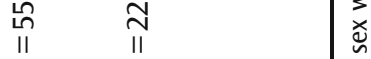

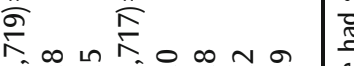

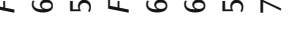

离

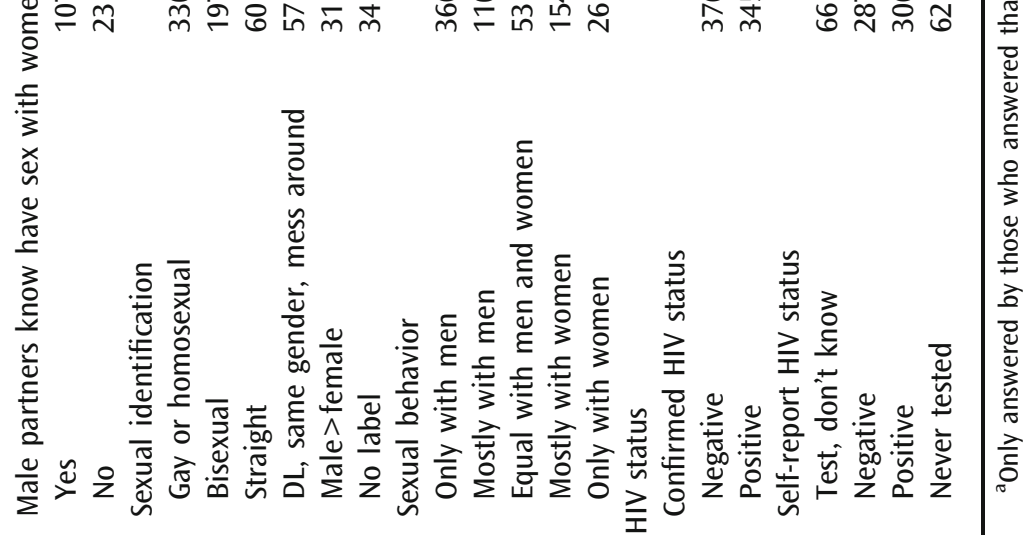




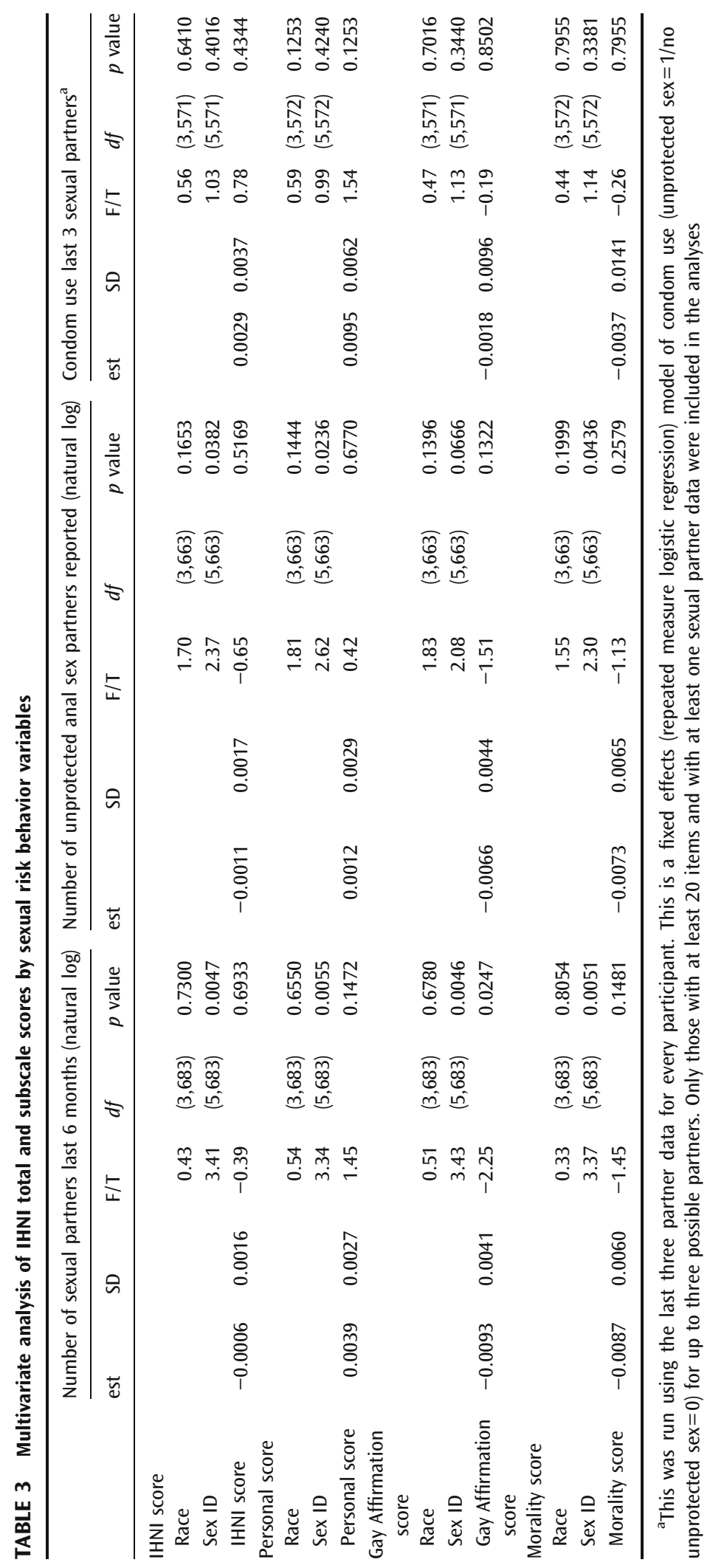


Table 3 shows that when the effects of ethnicity/race and sexual identification were controlled for, the Gay Affirmation subscale significantly and inversely predicted the number of sexual partners over the past 6 months. Sexual identification significantly predicted the number of AI partners. There were no associations between IHNI scores and use of condoms. These analyses adjusted for ethnicity/race and sexual identification but not the factor of MSM/MSM/W (sexual orientation in the past 6 months) due to collinearity. Only the Gay Affirmation subscale correlated significantly with number of sexual partners in the previous 6 months when controlling for race and self sexual identification, with higher Gay Affirmation scores associating with fewer partners. Evaluation of the coefficients in this model showed this effect significantly pronounced for the men who identified as straight $(-0.42, \mathrm{SE}=0.19 ; t=-2.18 ; p=0.0295)$, with very high Gay Affirmation scores and few sexual partners.

\section{DISCUSSION}

Findings showed the IHNI scale to have adequate psychometric properties and similar factor structure to the scale's original sample ${ }^{2}$. IHNI scores averaged ten points or more higher than the original sample, which suggests aspects of internalized homonegativity are more pronounced in poor, urban MSM and MSM/W of color than white middle-class, Midwest gay men. The IHNI total score and the three subscales captured distinct aspects of internalized homonegativity and described meaningful differences along meaningful participant characteristics, including social indicators of the participant's "outness." Levels of internalized homonegativity increased with age, with lower educational levels, with African American ethnicity, with experiences of poverty and homelessness, with recent use of cocaine (lower levels of homonegativity with methamphetamine use), with experiences of being incarcerated, with being a man who is behaviorally bisexual, and with being HIV seronegative.

The sampling frame involving RDS did not promote immediate convergence of IHNI scores for participants enrolled in the first versus the latter halves of each Wave of data collection. In both Waves of data collection, enrollment of African Americans (and concomitant higher IHNI scores) increased as the linked referrals proceeded. IHNI scores for each Wave, however, were similar. This application of RDS did not yield a sample that could be considered representative of the general population of MSM or MSM/W in Los Angeles County, particularly along the factors of ethnicity/race, poverty and HIV. Hence, findings are understood to reflect a unique sample of very poor MSM and MSM/W of color in Los Angeles County.

Drug-specific behaviors interacted with the IHNI scores and ethnicity/race such that African American MSM/W were more likely to have positive urine cocaine screens and higher IHNI scores, while White and Hispanic MSM were more likely to provide positive urine methamphetamine screens and lower IHNI scores. Although substance use is an efficient method to cover over feelings of internalized homophobia $^{17}$, its functions appear to be divergent for cocaine and for methamphetamine using men. Another distinction is the finding that African American men reported similar levels of drug use as White and Latino men, which contrasts with work showing lower levels of substance use in African American MSM/W ${ }^{15}$.

High IHNI scores for African American MSM/W validates the work of many and indicates that the sociocultural milieu of most African American men prohibit expressions of non-heterosexual behaviors and identities ${ }^{10,17,18}$. African American 
MSM/W may face potential rejection of cultural affiliation when openly acknowledging either male-male sexual behaviors or gay or bisexual identities ${ }^{7,19}$.

Consistent with prior work ${ }^{15}$, the highest homonegativity scores were reported by MSM/W who reportedly had no prior tests for HIV; HIV prevalence in this group was high. As such, homonegativity may function within this group of men as a barrier to HIV testing. Still, this sample of men with high homonegativity scores completed their rapid tests and learned their results. Design of prevention strategies with the goal of increasing HIV testing among men who have never tested may benefit from rapid testing procedures and/or monetary incentives.

IHNI scores generally did not predict HIV-related sexual risk behaviors after controlling for race/ethnicity and self sexual identification. One exception is that high scores on the Gay Affirmation subscale significantly predicted low numbers of sexual partners, particularly for men who self-identified as "straight." That only one model showed significant associations between IHNI scores and behavioral outcomes after holding race/ethnicity and self sexual identification constant indicates that there is no homogenous experience of sexual behaviors and internalized homonegativity for MSM and MSM/W of differing racial/ethnic groups who adhere to differing sexual identification labels.

Findings were limited by several factors. These include collecting all data from a single convenience sample in Los Angeles County and reliance primarily on subject reports. Yet, participants were scattered throughout the Los Angeles basin and comprised a coherent sample of predominantly low-income MSM and MSM/W of color. The size of the sample allowed sufficient design effect for findings to be considered significant, even if some participants misrepresented self-reports. As well, ACASI was used to increase privacy and findings comparing urine data with selfreport of drug use indicating participants approached the questionnaire straightforwardly. Finally, there is a limitation to the concept of internalized homonegativity that involves emphasis on individual pathology rather than on institutional/societal oppression $^{3}$.

Other limitations to these findings deserve mention that are related to the RDS method. In our use of dual cores of drug users and/or MSM in the RDS procedure, we compiled a sample that showed high levels of similarity between participants and the recruits they referred into the study (i.e., homophily) for most of the variables measured. These included HIV status, race/ethnicity, drug use, and levels of income, even though the sample was overwhelmingly poor. Implementation of RDS failed to yield a "representative" sample of drug users and/or MSM in both this and another RDS study in Los Angeles ${ }^{20}$, which also recruited a very poor sample with high HIV prevalence. This suggests findings should be constrained to similar urban groups of older MSM and MSM/W of color with high HIV prevalence and who are drug users.

Despite these limitations, findings still show internalized homonegativity to correlate significantly and strongly with a variety of demographic factors, drug use, sexual behaviors, and HIV status in this sample of very poor, largely minority MSM and MSM/W, which provide a rare glimpse into associations between internalized homonegativity, sexual behaviors, and drug use for the men in this understudied group. Findings also emphasize the value of using rapid testing procedures with those who do not know their HIV status and imply that optimally effective prevention interventions that address homonegativity and sexual risk may be constructed differently for MSM methamphetamine users than for MSM/W cocaine users. 


\section{ACKNOWLEDGEMENTS}

Drs. Shoptaw and Gorbach gratefully acknowledge the support of NIDA grant U01DA17394 for this work.

OPEN ACCESS This article is distributed under the terms of the Creative Commons Attribution Noncommercial License which permits any noncommercial use, distribution, and reproduction in any medium, provided the original author(s) and source are credited.

\section{REFERENCES}

1. HIV Epidemiology Program, Los Angeles County Department of Public Health. HIV/ AIDS Surveillance Summary, July 2008.

2. Mayfield W. The development of an Internalized Homonegativity Inventory for gay men. J Homosex. 2001; 41(2): 53-76. PMID: 11482428.

3. Williamson IR. Internalized homophobia and health issues affecting lesbians and gay men. Health Educ Res. 2000; 15(1): 97-107. Review. PMID: 10788206.

4. Stokes JP, Peterson JL. Homophobia, self-esteem, and risk for HIV among African American men who have sex with men. AIDS Educ Prev. 1998; 10(3): 278-292. PMID: 9642425.

5. Rosser BR, Metz ME, Bockting WO, Buroker T. Sexual difficulties, concerns, and satisfaction in homosexual men: an empirical study with implications for HIV prevention. J Sex Marital Ther. 1997; 23(1): 61-73. PMID: 9094037.

6. Wohl AR, Johnson DF, Lu S, et al. HIV risk behaviors among African American men in Los Angeles County who self-identify as heterosexual. J Acquir Immune Defic Syndr. 2002; 31(3): 354-360. PMID: 12439213.

7. Dodge B, Jeffries WL 4th, Sandfort TG. Beyond the Down Low: sexual risk, protection, and disclosure among at-risk Black men who have sex with both men and women (MSMW). Arch Sex Behav. 2008; 37(5): 683-696. PMID: 18512140.

8. Kashubeck-West S., Szymanski DM. Risky sexual behavior in gay and bisexual men: internalized heterosexism, sensation seeking, and substance use. The Counseling Psychologist. 2008; 36(4): 595-614.

9. Zea MC, Reisen CA, Díaz RM. Methodological issues in research on sexual behavior with Latino gay and bisexual men. Am J Community Psychol. 2003; 31(3-4): 281-291. PMID: 12866685.

10. Millett G, Malebranche D, Mason B, Spikes P. Focusing on the "down low": bisexual black men, HIV risk and heterosexual transmission. J Natl Med Assoc. 2005; 97(7 Suppl): 52S-59S.

11. Cochran SD, Mays VM. Physical health complaints among lesbians, gay men, and bisexual and homosexually experienced heterosexual individuals: results from the California Quality of Life Survey. Am J Public Health. 2007; 97(11): 2048-2055. Epub 2007 Apr 26. PMID: 17463371.

12. Halkitis PN, Mukherjee PP, Palamar JJ. Longitudinal modeling of methamphetamine use and sexual risk behaviors in gay and bisexual men. AIDS Behav. 2008. PMID: 18661225

13. Larkins S, Reback CJ, Shoptaw S. HIV risk behaviors among gay male methamphetamine users: before and after treatment. J Gay Lesb Psychother. 2006; 10(3-4): 123-129.

14. Jimenez AD. Triple jeopardy: targeting older men of color who have sex with men. $J$ Acquir Immune Defic Syndr. 2003; 33(Suppl 2): S222-S225.

15. Millett G, Flores SA, Peterson JL, Bakeman R. Explaining disparities in HIV infection among Black and White men who have sex with men: a meta-analysis of HIV-risk behaviors. AIDS. 2007; 21(15): 2083-2091. 
16. Heckathorn DD. Respondent-driven sampling: a new approach to the study of hidden populations. Social Problems. 1997; 44: 174-199.

17. Mays VM, Cochran SD, Zamudio A. HIV prevention research: are we meeting the needs of African American men who have sex with men. J Black Psychol. 2004; 30: 78-105.

18. Operario D, Smith CD, Kegeles S. Social and psychological context for HIV risk in nongay-identified African American men who have sex with men. AIDS Educ Prev. 2008; 20 (4): 347-359. PMID: 18673067.

19. Malebranche DJ. Black men who have sex with men and the HIV epidemic: next steps for public health. Am J Public Health. 2003; 93(6): 862-865. No abstract available. PMID: 12773340.

20. Marks G, Millett GA, Bingham T, et al. Understanding differences in HIV sexual transmission among Latino and Black men who have sex with men: The Brothers y Hermanos Study [published online ahead of print August 8, 2008]. AIDS Behav. 